\title{
Non-Holonomic Control I
}

\author{
E. Brion \\ V.M. Akulin \\ Laboratoire Aimé Cotton, Laboratoire Aimé Cotton, \\ CNRS II, Bâtiment 505, CNRS II, Bâtiment 505, \\ 91405 Orsay Cedex, France. 91405 Orsay Cedex, France. \\ D. Comparat \\ I. Dumer \\ Laboratoire Aimé Cotton, College of Engineering, \\ CNRS II, Bâtiment 505, University of California, \\ 91405 Orsay Cedex, France. Riverside, CA 92521, USA. \\ V. Gershkovich \\ Institut des Hautes Etudes Scientifiques, \\ Bures-sur-Yvette, France. \\ G. Harel \\ Department of Computing, \\ University of Bradford, \\ Bradford, West Yorkshire BD'7 1DP, United Kingdom. \\ G. Kurizki \\ Department of Chemical Physics, \\ Weizmann Institute of Science, \\ 76100 Rehovot, Israel. \\ I. Mazets \\ Department of Chemical Physics, \\ Weizmann Institute of Science, \\ 76100 Rehovot, Israel. \\ A.F. Ioffe Physico-Technical Institute, \\ 194021 St. Petersburg, Russia. \\ P. Pillet \\ Laboratoire Aimé Cotton, \\ CNRS II, Bâtiment 505, \\ 91405 Orsay Cedex, France.
}

November 11, 2018 


\begin{abstract}
In this paper, we present a universal control technique, the non-holonomic control, which allows us to impose any arbitrarily prescribed unitary evolution to any quantum system through the alternate application of two well-chosen perturbations.
\end{abstract}

\title{
1 Introduction
}

Quantum control is very topical and interests many different fields of contemporary physics and chemistry, such as Molecular Dynamics in laser fields and Quantum Optics 1, 2, 3, 4, 5. A few examples of control of the quantum state by conditional measurements [6, 7], by adiabatic transport or by unitary evolution have been already proposed for the particular quantum system of atoms interacting with quantized electromagnetic field in a single-mode resonator. In parallel, a theoretical framework of quantum control has been built up : in particular, several classes of problems have been distinguished such as the control of quantum evolution, quantum state, or density matrix ; besides, the mathematical necessary conditions for their feasibility have been derived in the context of the theory of Lie groups $[8,9$. Moreover, theoretical methods, mainly based on optimization techniques, have been designed to exhibit the explicit values of the control parameters.

Most of these traditional approaches rely either on a known or intuitively guessed particular solution which can be further optimized with respect to a given cost functional, through variational schemes [10. By contrast, identifying the convergence domain of the standard algorithms through straightforward exploration of the entire multidimensional space of the control parameters implies numerical work, the complexity of which grows exponentially with the dimensionality of the system. Though, as we shall see in this paper, an original method, called non-holonomic bang-bang control [11, solves this problem for complex enough quantum systems so that no separable subsystems can be singled out : in terms of classical mechanics, such systems do not have holonomic constraints. The physical idea is to alternately apply two distinct perturbations $\widehat{P}_{a}$ and $\widehat{P}_{b}$ during pulses, the timings of which play the role of control parameters and are determined by solving the "inverse Floquet problem". Equivalently, one can fix the duration of the pulses and choose the strength of the perturbations as the free parameters one has to adjust in order to achieve the control objective. Actually, the convergence of our algorithm results from an unsuspected simplification emerging from the Random Matrix Theory. Indeed, it relies on the algebraic properties of the $N^{t h}$ roots of the identity matrix, the spectra of which resemble to those of random unitary matrices which obey the Dyson distribution law.

This paper is organized as follows. In the first part, we recall the broad context of the theory of quantum control. In the second part, we present the non-holonomic control technique, and provide all the algorithmic tools which allow one to implement it. 


\section{Quantum Control}

Quantum control is a very topical issue in contemporary physics. The needs for control are particularly sensitive in Molecular Dynamics and Quantum Optics, and are quite various : indeed, one should not speak of quantum control, but rather of quantum controls. Actually, four different types of problems have been identified in the literature 10, 12]: the control of pure state, the control of density matrix, the control of observable and, finally, the control of the evolution operator. For each of these problems, the goal is the same, that is to impose the considered characteristics an arbitrarily chosen value.

To achieve a control objective, one has to perturb the system, since its natural evolution usually results in too restrictive a dynamics. The control Hamiltonian $\widehat{H}(t)$ comprises thus the unperturbed Hamiltonian $\widehat{H}_{0}$ as well as $M$ Hamiltonians of the form $C_{m}(t) \widehat{P}_{m}$, which can stand for the interaction Hamiltonians of the system with $M$ classical fields, the amplitudes of which, denoted by $C_{m}(t)$, are controlled by an external operator

$$
\widehat{H}(t)=\widehat{H}_{0}+\sum C_{m}(t) \widehat{P}_{m} .
$$

The functions $\left\{C_{m}(t)\right\}$ play the role of the control parameters one has to adjust in order to achieve the desired control process. In other words, any problem of control can always be translated into the following form : for the physical system considered, perturbed through given fields of controllable amplitudes $\left\{C_{m}(t)\right\}$, one looks for the values of the control paramaters $\left\{C_{m}(t)\right\}$ such that a specific characterics (quantum state, density matrix, observable, evolution operator) takes an arbitrarily prescribed value.

All the objectives are not feasible. Some of them are forbidden by the unitarity of the evolution operator for closed systems : for example, one cannot change the eigenvalues of the density matrix through a Hamiltonian process of control. This kind of constraints is often referred to as kinematical constraints 13. But there also exist dynamical constraints which stem from the algebraic properties of the Hamiltonians $\left\{\widehat{P}_{m}\right\}$. Indeed, the evolution operator

$$
\widehat{U}(t)=\mathcal{T}\left\{e^{-\frac{i}{\hbar} \int_{0}^{t} \widehat{H}(\tau) d \tau}\right\}
$$

where $\mathcal{T}$ denotes the chronological product, belongs to the Lie group obtained by exponentiation of the Lie algebra generated by the operators $\left\{i \widehat{H}_{0}, i \widehat{P}_{m}\right\}$. So, it appears clearly that the feasibility of a particular problem of control in a specific physical situation, defined by the Hamiltonians $\left\{\widehat{P}_{m}\right\}$, is closely related to the properties of this algebra : for example, if one wants to completely control the evolution operator of a quantum system, that is if one wants to be able to give the operator $\widehat{U}$ any prescribed value, one must perturb it in such a way that the operators $\left\{i \widehat{H}_{0}, i \widehat{P}_{m}\right\}$ generate the whole Lie algebra $u(N)$ which provides, through exponentiation, the whole Lie group $U(N)$ [8, 14]. Necessary 
mathematical conditions also exist for the other types of control problems and can be found in literature 12] : these conditions are obviously weaker than the previous one, since the evolution controllability automatically implies all the other ones.

The feasibility of a control problem can thus be decided through mathematical criteria established in the context of the Lie group theory. But the explicit values of the control parameters achieving the desired control objective still remain to be found. In other words, once the existence of a solution has been proved, one has to find it explicitly. Different methods have been proposed, such as optimal control [15, 16, 2, which mainly employ optimization techniques : the idea is to write down a functional the optimization of which gives the best compromise between all the constraints. A purely algebraic approach [17, based on the decomposition of the arbitrary desired evolution on the Lie group, is also possible, but rapidly leads to intractable computations as the dimension of the state space increases.

In the next section, we describe a universal control technique, called nonholonomic control [1], which allows one to control the evolution operator of an arbitrary quantum system through the alternate applications of two wellchosen perturbations. Two equivalent sets of control parameters are possible : one can choose to freeze the amplitudes of the perturbations and let the pulse timings play the role of free parameters, or, on the contrary, freeze the pulse timings and adjust the amplitudes on each control pulse. We shall present the algorithm which allows us to compute the explicit values of control parameters, and emphasize the algebraic reasons which assure its convergence.

\section{Control of the evolution through non-holonomic control}

Let us consider an $N$-dimensional quantum system of unperturbed Hamiltonian $\widehat{H}_{0}$. Our goal is to control its evolution operator $\widehat{U}$, i.e. to be able to achieve any arbitrary evolution $\widehat{U}_{\text {arbitrary }} \in U(N)$. To this end, we alternately apply two physical perturbations, of Hamiltonians $\widehat{P}_{a}$ and $\widehat{P}_{b}$, during $N^{2}$ pulses the timings of which are denoted by $\left\{\tau_{k} \equiv t_{k}-t_{k-1}\right\}_{k=1, \ldots, N^{2}}\left(t_{0}=0\right.$ and $t_{N^{2}}=T$ correspond to the beginning and the end of the control sequence, respectively) : the total Hamiltonian takes the following pulsed shape (cf fig. 1)

$$
\widehat{H}(t)=\widehat{H}_{0}+C_{a}(t) \widehat{P}_{a}+C_{b}(t) \widehat{P}_{b}
$$

where

$$
\begin{aligned}
& C_{a}(t)=1, C_{b}(t)=0 \text { and } \widehat{H}(t)=\widehat{H}_{0}+\widehat{P}_{a} \equiv \widehat{H}_{a} \text { for } t \in\left[t_{2 k}, t_{2 k+1}\right] \\
& C_{a}(t)=0, C_{b}(t)=1 \text { and } \widehat{H}(t)=\widehat{H}_{0}+\widehat{P}_{b} \equiv \widehat{H}_{b} \text { for } t \in\left[t_{2 k-1}, t_{2 k}\right] \\
& \text { for } k=1, \ldots, N^{2},
\end{aligned}
$$




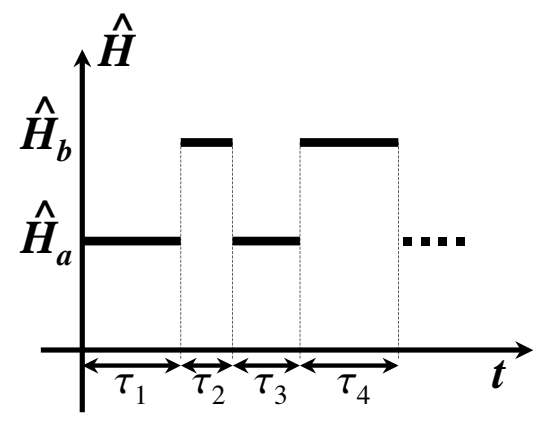

Figure 1: Pulsed shape of the control Hamiltonian.

and the total evolution operator is

$$
\widehat{U}\left(\left\{\tau_{1}, \ldots, \tau_{N^{2}-1}, \tau_{N^{2}}\right\}\right)=e^{-\frac{i}{\hbar} \widehat{H}_{b} \tau_{N^{2}}} \cdot e^{-\frac{i}{\hbar} \widehat{H}_{b} \tau_{N^{2}-1}} \ldots e^{-\frac{i}{\hbar} \widehat{H}_{a} \tau_{1}} .
$$

where we have implicitly assumed that $N$ is even.

Our control problem can thus be translated into the following form : given $\widehat{U}_{\text {arbitrary }} \in U(N)$, an arbitrary unitary operator, we want to find a time vector $\vec{\tau}=\left(\begin{array}{c}\tau_{1} \\ \vdots \\ \tau_{N^{2}-1} \\ \tau_{N^{2}}\end{array}\right)$ such that

$$
\widehat{U}(\vec{\tau})=\widehat{U}_{\text {arbitrary }}
$$

As we said previously, for a solution to exist the operators $\left\{i \widehat{H}_{a}, i \widehat{H}_{b}\right\}$ must generate the whole Lie algebra $u(N)$ : this is called the "bracket generation condition". This property can be checked directly as long as the dimension $N$ is not too big : one simply computes the commutators of all orders of $i \widehat{H}_{a}$ and $i \widehat{H}_{b}$ and stops as soon as they generate $u(N)$. But when $N$ becomes large, direct computation is intractable. In that case, one can simply check the following sufficient condition, suggested by V. Kac, according to which the system becomes non-holonomic, that is completely controllable, when the representative matrix of $\widehat{H}_{b}$ in the eigenbasis of $\widehat{H}_{a}$ has no off-diagonal zeros.

Once the previous criterion is checked, one has to compute the time vector $\vec{\tau}$ solution of Eq.(11). The method consists first in determining the time vector $\vec{\tau}(0)$ such that

$$
\widehat{U}\left(\vec{\tau}^{(0)}\right)=\widehat{I},
$$

and then iteratively approaching the time vector $\vec{\tau}$ through a Newton-like technique. 
The straightforward way to compute $\vec{\tau}^{(0)}$ would be to minimize the functional

$$
F(\vec{\tau})=\|\widehat{U}(\vec{\tau})-\widehat{I d}\|^{2}
$$

with respect to $\vec{\tau}$. However, $F$ presents many local minima which make its optimization uneasy. Nevertheless there exists an alternative method based on the algebraic properties of the $N^{t h}$ roots of the identity matrix. The idea is to look for $N$ parameters $\left\{T_{k}\right\}_{k=1 \ldots N}$ such that

$$
e^{-\frac{i}{\hbar} \widehat{H}_{b} T_{N}} \cdot e^{-\frac{i}{\hbar} \widehat{H}_{a} T_{N-1}} \ldots e^{-\frac{i}{\hbar} \widehat{H}_{a} T_{1}}=\widehat{I}^{\frac{1}{N}},
$$

where $\widehat{I}^{\frac{1}{N}}$ is an $N^{t h}$ root of the identity matrix, i.e. a matrix the eigenvalues of which coincide with the $N^{t h}$ roots of the unity $\left\{e^{i k \frac{2 \pi}{N}}\right\}_{k=0, \ldots,(N-1)}$; in other words, $\widehat{I}^{\frac{1}{N}}$ is a matrix of the form

$$
\widehat{I}^{\frac{1}{N}}=\widehat{M}^{-1} \cdot\left[\begin{array}{cccc}
1 & 0 & \cdots & 0 \\
0 & e^{i \frac{2 \pi}{N}} & \cdots & 0 \\
\vdots & \vdots & \ddots & \vdots \\
0 & 0 & \cdots & e^{i(N-1) \frac{2 \pi}{N}}
\end{array}\right] \cdot \widehat{M}
$$

where $\widehat{M}$ is a unitary matrix. To compute the $T_{k}$ 's, we use the following algebraic property : if $P_{\widehat{U}}(\lambda) \equiv \sum_{j=0}^{N} a_{j} \lambda^{j}$ denotes the characteristic polynomial of a unitary matrix $\widehat{U}$, then $\sum_{j=0}^{N}\left|a_{j}\right|^{2} \geq 2$ and the equality is achieved iff $\widehat{U}$ is an $N^{t h}$ root of the identity matrix, up to a global phase factor. To obtain the $T_{k}$ 's, one thus computes the characteristic polynomial $P(\lambda) \equiv \sum_{j=0}^{N} a_{j}\left(\left\{T_{k}\right\}_{k=1 \ldots N}\right) \lambda^{j}$ of the matrix product

$$
e^{-\frac{i}{\hbar} \widehat{H}_{b} T_{N}} \cdot e^{-\frac{i}{\hbar} \widehat{H}_{a} T_{N-1}} \ldots e^{-\frac{i}{\hbar} \widehat{H}_{a} T_{1}},
$$

and minimizes the function $F_{N}=\sum_{j=0}^{N}\left|a_{j}\left(\left\{T_{k}\right\}_{k=1 \ldots N}\right)\right|^{2}$ to 2 with respect to the $T_{k}$ 's. This minimization turns to be quite easy, due to the fact that a generic unitary matrix is very close to an $N^{t h}$ root of the identity. In fact, numerical work shows that in about $30 \%$ cases of randomly chosen timings $\left\{T_{k}\right\}$ the standard steepest descent algorithm immediatly finds the global minimum $F_{N}=2$. This fact has roots in the Random Matrix Theory. Indeed, according to Dyson's law, the eigenvalues of random unitary matrices tend to repel each other, and are thus very likely to be almost regularly distributed on the unit circle, as those of an $N^{t h}$ root of the identity, as shown in Fig 2 In other words, in the space of $N \times N$ unitary matrices, the $\widehat{I}^{\frac{1}{N}}$ matrices are present in abundance, and can be reached from randomly chosen point by small variation of the timings.

Finally, we define the time vector $\vec{\tau}^{(0)}$ corresponding to the identity matrix by simple repetition of $\left\{T_{k}\right\}$

$$
\tau_{i+(j-1) N}^{(0)}=T_{i} \text { for } i, j=1, \ldots, N,
$$




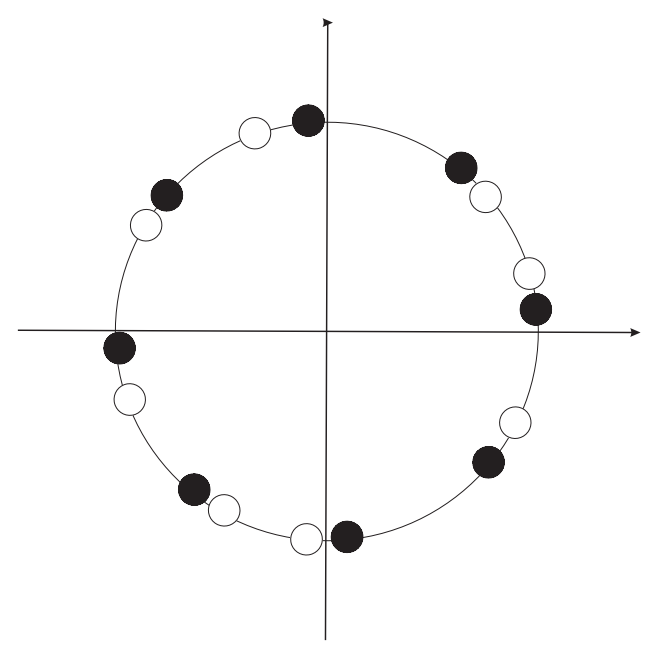

Figure 2: Spectrum of a random unitary matrix (white circles) resulting from the repulsion of the eigenvalues on a unit circle is shown vs the eigenvectors of $N^{t h}$ root of the identity matrix (black circles) multiplied by an unimportant phase factor.

and checks that indeed

$$
\begin{aligned}
\widehat{U}\left(\vec{\tau}^{(0)}\right) & =\underbrace{\underbrace{e^{-\frac{i}{\hbar} \widehat{H}_{b} T_{N}} \cdot e^{-\frac{i}{\hbar} \widehat{H}_{a} T_{N}} \ldots e^{-\frac{i}{\hbar} \widehat{H}_{a} T_{N}}}_{\widehat{I}^{\frac{1}{N}}} \cdots \underbrace{e^{-\frac{i}{\hbar} \widehat{H}_{b} T_{N}} \cdot e^{-\frac{i}{\hbar} \widehat{H}_{a} T_{N}} \ldots e^{-\frac{i}{\hbar} \widehat{H}_{a} T_{N}}}_{\widehat{I}^{\frac{1}{N}}}}_{N \text { times }} \\
& =\widehat{I}
\end{aligned}
$$

up to an unimportant global phase factor.

We now have to iteratively determine the time vector $\vec{\tau}$ from $\vec{\tau}^{(0)}$. Let us first consider the case of a target evolution close to the identity : in that case, $\widehat{U}_{\text {arbitrary }}$ can be written under the form

$$
\widehat{U}_{\text {arbitrary }}=\widehat{U}_{\epsilon} \equiv \exp (-i \widehat{\mathcal{H}} \epsilon),
$$

where $\widehat{\mathcal{H}}$ is an $8 \times 8$ bounded $(\|\widehat{\mathcal{H}}\| \leq 1)$ dimensionless Hermitian Hamiltonian, and $\epsilon>0$ a small parameter. We then look for the variations $\delta \tau_{k}$, determined to first order in $\epsilon$ by the linear equations

$$
\sum_{k=1}^{N^{2}} \frac{\partial \widehat{U}}{\partial \tau_{k}}\left(\vec{\tau}^{(0)}\right) \delta \tau_{k}=-i \widehat{\mathcal{H}} \epsilon .
$$

Once $\delta \vec{\tau}$ has been calculated through standard techniques of linear algebra, we replace $\vec{\tau}(0)$ by $\vec{\tau}^{(0)}+\delta \vec{\tau}$ and repeat the same operation until we obtain $\vec{\tau}$ which checks $\widehat{U}(\vec{\tau})=\widehat{U}_{\text {arbitrary }}$ at the desired accuracy. 
If the evolution $\widehat{U}_{\text {arbitrary }}=\widehat{U}_{\epsilon}$ is not close to the identity, that is if $\epsilon$ is not small, one has to divide the work into elementary paths on which the previous method converges. To this end, we consider an integer $n \geq 2$ such that $\left(\widehat{U}_{\text {arbitrary }}\right)^{\frac{1}{n}}=\widehat{U}_{\frac{\epsilon}{n}}$ is attainable from $\widehat{I}$ through our iterative algorithm, and determine in this way the associated time vector $\vec{\tau}_{\left(\frac{1}{n}\right)}$ which checks

$$
\widehat{U}\left(\vec{\tau}_{\left(\frac{1}{n}\right)}\right)=\widehat{U}_{\frac{\epsilon}{n}}
$$

Taking $\left(\widehat{U}_{\text {arbitrary }}\right)^{\frac{1}{n-1}}=\widehat{U}_{\frac{\epsilon}{n-1}}$ as our new target, we repeat the same algorithm to compute $\vec{\tau}\left(\frac{1}{n-1}\right)$ such that

$$
\widehat{U}\left(\vec{\tau}_{\left(\frac{1}{n-1}\right)}\right)=\widehat{U}_{\frac{\epsilon}{n-1}},
$$

and so on. We progress in this way as long as our algorithm converges : in general, it stops at a value $n^{*} \geq 1$, for which the system Eq(5) has no solution. Then, we keep the time vector $\vec{\tau}\left(\frac{1}{n^{*}}\right)$ and simply repeat the same control sequence $n^{*}$ times to achieve the desired evolution

$\underbrace{\widehat{U}\left(\vec{\tau}_{\left(\frac{1}{n^{*}}\right)}\right) \cdot \widehat{U}\left(\vec{\tau}_{\left(\frac{1}{n^{*}}\right)}\right) \ldots \widehat{U}\left(\vec{\tau}_{\left(\frac{1}{n^{*}}\right)}\right)}_{n^{*} \text { times }}=\left(\widehat{U}_{\frac{\epsilon}{n^{*}}}\right)^{n^{*}}=\left[\left(\widehat{U}_{\text {arbitrary }}\right)^{\frac{1}{n^{*}}}\right]^{n^{*}}=\widehat{U}_{\text {arbitrary }}$.

To conclude this section, we provide an equivalent form of our method. Indeed, in the previous paragraphs we fixed the amplitudes of the perturbations once for all and considered the pulse timings $\tau_{k}$ as our free control parameters. But we also might have chosen to apply $N^{2}$ pulses of same duration $\tau=\frac{T}{N^{2}}$, where $T$ is the total control sequence duration, and taken the amplitudes as our free control variables. In other words, we might have applied the following Hamiltonian

$$
\widehat{H}(t)=\left\{\begin{aligned}
\widehat{H}_{2 k+1} & =\widehat{H}_{0}+C_{2 k+1} \widehat{P}_{a} \\
\widehat{H}_{2 k} & =\widehat{H}_{0}+C_{2 k} \widehat{P}_{b}
\end{aligned}\right.
$$

where the amplitudes $\left\{C_{k}\right\}_{k=1, \ldots . N^{2}}$ play the role of adjustable control parameters. The evolution operator would have thus taken the form

$$
\widehat{U}\left(\left\{C_{k}\right\}\right)=e^{-\frac{i}{\hbar}\left(\widehat{H}_{0}+C_{N^{2}} \widehat{P}_{b}\right) \tau} \cdot e^{-\frac{i}{\hbar}\left(\widehat{H}_{0}+C_{N^{2}-1} \widehat{P}_{a}\right) \tau} \ldots e^{-\frac{i}{\hbar}\left(\widehat{H}_{0}+C_{1} \widehat{P}_{a}\right) \tau},
$$

and our problem of control would have boiled down to finding the vector $\vec{C}=$ $\left(\begin{array}{c}C_{1} \\ \vdots \\ C_{N^{2}-1} \\ C_{N^{2}}\end{array}\right)$ such that $\widehat{U}(\vec{C})=\widehat{U}_{\text {arbitrary }}$. 
The method remains the same as previously. First, one determines the parameters $\left\{c_{k}\right\}_{k=1, \ldots, N}$ such that

$$
e^{-\frac{i}{\hbar}\left(\widehat{H}_{0}+c_{N} \widehat{P}_{b}\right) \tau} \cdot e^{-\frac{i}{\hbar}\left(\widehat{H}_{0}+c_{N-1} \widehat{P}_{a}\right) \tau} \ldots e^{-\frac{i}{\hbar}\left(\widehat{H}_{0}+c_{1} \widehat{P}_{a}\right) \tau}=\widehat{I}^{\frac{1}{N}},
$$

by minimizing the functional $\sum_{j=0}^{N}\left|a_{j}\left(\left\{c_{k}\right\}_{k=1 \ldots N}\right)\right|^{2}$ to 2 , where $\left\{a_{j}\right\}$ denote the coefficients of the characteristic polynomial of the matrix product

$$
e^{-\frac{i}{\hbar}\left(\widehat{H}_{0}+c_{N} \widehat{P}_{b}\right) \tau} \cdot e^{-\frac{i}{\hbar}\left(\widehat{H}_{0}+c_{N-1} \widehat{P}_{a}\right) \tau} \ldots e^{-\frac{i}{\hbar}\left(\widehat{H}_{0}+c_{1} \widehat{P}_{a}\right) \tau},
$$

and we set

$$
C_{i+(j-1) N}^{(0)}=c_{i} \text { for } i, j=1, \ldots, N,
$$

such that $\widehat{U}\left(\vec{C}^{(0)}\right)=\widehat{I}$.

Then, one iteratively approaches the desired vector $\vec{C}$ in the same way as previously. For a target evolution $\widehat{U}_{\text {arbitrary }}=\widehat{U}_{\epsilon}=\exp (-i \widehat{\mathcal{H}} \epsilon)$ close to the identity (i.e. for $\epsilon$ small), one computes the variations $\delta C_{k}$ to first order in $\epsilon$ by solving the linear equations

$$
\sum_{k=1}^{N^{2}} \frac{\partial \widehat{U}}{\partial C_{k}}\left(\vec{C}^{(0)}\right) \delta C_{k}=-i \widehat{\mathcal{H}} \epsilon .
$$

Then we replace $\vec{C}^{(0)}$ by $\vec{C}^{(0)}+\delta \vec{C}$ and repeat the same operation, and so on, until we get $\vec{C}$ which checks $\widehat{U}(\vec{C})=\widehat{U}_{\text {arbitrary }}$ with the desired accuracy.

For a target evolution $\widehat{U}_{\text {arbitrary }}=\widehat{U}_{\epsilon}$ far from the identity (i.e. for $\epsilon$ finite), one considers an arbitrary integer $n \geq 1$ such that $\left(\widehat{U}_{\text {arbitrary }}\right)^{\frac{1}{n}}=\widehat{U}_{\frac{\epsilon}{n}}$ can be reached from the identity through the previous iterative algorithm, and computes $\vec{C}\left(\frac{1}{n}\right)$ which checks

$$
\widehat{U}\left(\vec{C}^{\left(\frac{1}{n}\right)}\right)=\widehat{U}_{\frac{\epsilon}{n}} .
$$

Then, one calculates in the same way the vector $\vec{C}\left(\frac{1}{n-1}\right)$ such that

$$
\widehat{U}\left(\vec{C}^{\left(\frac{1}{n-1}\right)}\right)=\widehat{U}_{\frac{\epsilon}{n-1}},
$$

and so on, until one obtains the limiting value $n^{*} \geq 1$ beyond which the algorithm fails to converge. Finally, one gets the desired evolution by repeating the same control sequence $n^{*}$ times, the amplitudes of which are given by the vector $\vec{C}\left(\frac{1}{n^{*}}\right)$.

\section{Conclusion}

In this paper, we presented a universal method for controlling the evolution operator of any quantum system. This control scheme, called the non-holonomic 
control, consists in alternately applying two physical perturbations which check the bracket generation condition. The role of tunable control parameters may be played either by the durations of the interaction pulses or by the strengths of the perturbations during these pulses. The calculation of the control parameters is achieved through an algorithm, that we presented here, the convergence of which is assured by remarkable properties of the $N^{t h}$ roots of the identity matrix.

In the following paper, we show how the non-holonomic control technique can be used in the context of quantum computation in order to build controlled quantum devices.

\section{References}

[1] D. J. Tannor and S. A. Rice, J. Chem. Phys. 83, 5013 (1985); D. J. Tannor, R. Kosloff, and S. A. Rice, J. Chem. Phys. 85, 5805 (1986).

[2] J.P. Palao and R. Kosloff, Phys. Rev. Lett. 89, 188301 (2002).

[3] M. Shapiro and P. Brumer, J. Chem. Phys. 84, 4103 (1986); P. Brumer and M. Shapiro, Chem. Phys. Lett. 126, 54 (1986).

[4] A. P. Peirce, M. A. Dahleh, and H. Rabitz, Phys. Rev. A 37, 4950 (1988); R. S. Judson and H. Rabitz, Phys. Rev. Lett. 68, 1500 (1992); V. Ramakrishna, M. V. Salapaka, M. Dahleh, H. Rabitz, and A. Peirce, Phys. Rev. A 51, 960 (1995); V. Ramakrishna and H. Rabitz, Phys. Rev. A 54, 1715 (1996).

[5] C. K. Law and J. H. Eberly, Phys. Rev. Lett. 76, 1055 (1996).

[6] K. Vogel, V. M. Akulin, and W. P. Schleich, Phys. Rev. Lett. 71, 1816 (1993).

[7] G. Harel, G. Kurizki, J.K. McIver, and E. Coutsias, Phys. Rev. A 53, 4534 (1996).

[8] V. Jurdjevic and H. Sussman, J. Differential Equations 12 (1972), 313.

[9] G. M. Huang, T. J. Tarn, and J. W. Clark, J. Math Phys. 24, 2608 (1983).

[10] A.G. Butkovskiy and Yu.I. Samoilenko, "Control of Quantum-Mechanical Processes and Systems"', Kluwer Academic Publishers, Dordrecht (Netherlands), 1990.

[11] G. Harel and V. M. Akulin, Phys. Rev. Lett. 82, 1 (1999).

[12] S.G. Schirmer, I.C.H. Pullen and A.I. Solomon, Hamiltonian and Lagrangian Methods in Nonlinear Control (Elsevier Science Ltd, 2003), quant-ph/0302121. 
[13] S.G. Schirmer, A.I. Solomon and J.V. Leahy, J. Phys. A 35, 4125-4141 (2002); S.G. Schirmer, A.I. Solomon and J.V. Leahy, J. Phys. A 35, 8551$8562(2002)$.

[14] V. Ramakrishna, M.V. Salapaka, M. Dahleh, H. Rabitz and A. Peirce, Phys. Rev. A 51, 960 (1995).

[15] Y. Ohtsuki, H. Kono and Y. Fujimura, J. Chem. Phys. 109 (21), 9318-31 (1998).

[16] S.G. Schirmer, M.D. Girardeau and J.V. Leahy, Phys.Rev. A 61, 012101 (2000).

[17] S.G. Schirmer, A.D. Greentree, V. Ramakrishna and H. Rabitz, quant-ph/0105155 (2001). 